\title{
WZ Cas - variability on multiple time-scales
}

\author{
T. Lebzelter ${ }^{1}$, R. F. Griffin ${ }^{2}$, and K. H. Hinkle ${ }^{3}$ \\ ${ }^{1}$ Institute for Astronomy (IfA), University of Vienna, Türkenschanzstrasse 17, 1180 Vienna, Austria \\ e-mail: lebzelter@astro.univie.ac.at \\ 2 The Observatories, Madingley Road, Cambridge CB3 OHA, UK \\ 3 National Optical Astronomy Observatory ${ }^{\star}$, PO Box 26732, Tucson, AZ 85726, USA
}

Received 16 March 2005 / Accepted 23 May 2005

\begin{abstract}
We present results on our long-term velocity monitoring of the C-rich semi-regular variable WZCas with a Coravel instrument. Our findings are compared with light changes of the star. We suggest that the two main periods, 366 and $186 \mathrm{~d}$, are due to radial pulsation. Furthermore, we find an interesting variation of the width and depth of the cross-correlation profile over a time-scale of at least $1000 \mathrm{~d}$. Several possible explanations for this behaviour are discussed by comparison of time-scales and expected variability amplitudes. The influence of movements of large convective cells on the line profiles seems to be the most likely explanation of some of the observed phenomena.
\end{abstract}

Key words. stars: late-type - stars: AGB and post-AGB - stars: variables: general

\section{Introduction}

The bright carbon star WZCas is a semi-regular variable (SRV). The star is a well known member of the carbon star class, with an extremely strong 16708 - $\AA$ Li line (Keenan 1993) and strong ${ }^{13} \mathrm{C}$ features (Herbig 1955). The large Li (e.g. Abia et al. 1999) and ${ }^{13} \mathrm{C}$ abundances in WZ Cas are the result of hot bottom burning, indicating that WZ Cas is a relatively more massive carbon star. As a second indication of its rather massive nature, Abia \& Isern (2000) find a high luminosity of $M_{\mathrm{bol}}=-6.44$. It is the most luminous star in their sample of local C-stars. A more recent investigation by Bergeat et al. (2002), however, gives a somewhat lower value of $M_{\mathrm{bol}}=-5.48$.

The $\mathrm{C} / \mathrm{O}$ ratio of $\mathrm{WZCas}$ is close to 1 , so Abia \& Isern (2000) classify the star as SC. In their detailed study of carbon star abundances, Lambert et al. (1986) find ${ }^{12} \mathrm{C} /{ }^{13} \mathrm{C}=4.5$ and $\mathrm{C} / \mathrm{O}=1.01$. The star turned out to be very rich in ${ }^{13} \mathrm{C}$ compared to other bright galactic field carbon stars analysed by Lambert et al. This is again probably due to hot bottom burning converting ${ }^{12} \mathrm{C}$ into ${ }^{13} \mathrm{C}$ and ${ }^{14} \mathrm{~N}$, which is a further indicator for the high mass of WZ Cas (Lambert et al. 1986). Keenan (1993) did not include WZ Cas among the J-type carbon stars, owing to its abundance peculiarities.

Richichi \& Percheron (2002) report a (limb-darkened) angular diameter of 7.11 mas. The Hipparcos parallax is $1.27 \pm 0.70$ mas; taking it at face value we can estimate an

* Operated by the Association of Universities for Research in Astronomy, Inc. under cooperative agreement with the National Science Foundation. approximate diameter of $600 R_{\odot}$. Owing to the large uncertainty of the distance, that value has to be seen only as an estimate. Typical radii of Asymptotic Giant Branch (AGB) stars are 200 to $800 R_{\odot}$ (e.g. van Belle et al. 2002), so $600 R_{\odot}$ seems to be of the correct order of magnitude. Its effective temperature has been determined as $3000 \mathrm{~K}$ (see a summary of different literature values in Gautschy-Loidl et al. 2004). Its mass-loss rate of $6.5 \times 10^{-9} M_{\odot} \mathrm{yr}^{-1}$ is rather low for an evolved asymptoticgiant-branch (AGB) star (Schoier \& Olofsson 2001).

WZ Cas was included in a study of the periods of semiregular red variables by Kiss et al.(1999), who found that the star is bi-periodic with periods of 373 and $187 \mathrm{~d}$. Such multi-periodicity has been found in quite a number of SRVs. Barnbaum \& Hinkle (1995) presented a first study of velocity variations in WZCas. Four velocities measured from nearinfrared $\mathrm{CN}$ and $\mathrm{Ti}$ lines at different pulsation phases showed a range of $6 \mathrm{~km} \mathrm{~s}^{-1}$. A centre-of-mass velocity of $-34.3 \mathrm{~km} \mathrm{~s}^{-1}$ has been given by Olofsson et al. (1993). No further systematic studies on the variability of the star are found in the literature.

WZ Cas has been observed occasionally since 1983 with photoelectric radial-velocity instruments. Intensive and systematic monitoring was initiated when we set up a collaboration whereby photometry and radial velocities were to be measured in a coordinated fashion. Here we report almost 5 years' velocity monitoring of the star together with scattered older measurements, and compare the findings with changes in the light-curve.

This paper is structured as follows: after giving some basic information on the radial-velocity measurements (Sect. 2), we discuss in Sect. 4 the light-curve of the star on the basis of 
archival data complemented by our own photometric measurements (Sect. 3). A more general discussion ensues in Sect. 5.

\section{Radial-velocity measurements}

The radial velocity of WZ Cas has been measured since 1983, initially with the original photoelectric radial-velocity spectrometer at Cambridge (Griffin 1967), subsequently with the Haute-Provence (OHP) Coravel (Baranne et al. 1979) and occasionally with the spectrometers at the Dominion Astrophysical Observatory (Fletcher et al. 1982) and Palomar (Griffin \& Gunn 1974), and most recently and intensively with the Cambridge Coravel, no description of which has been published but which resembles in many ways the OHP one. These instruments use a wavelength range between 3600 and $5200 \AA$.

Velocity measurements have also been made from near-infrared spectra obtained with the Fourier Transform Spectrometer (FTS) at the Kitt Peak $4 \mathrm{~m}$ telescope and at the coudé-feed telescope at Kitt Peak with the NICMASS detector (Joyce et al. 1998). The FTS spectra were at resolution as high as 70000 and are reported on in Barnbaum \& Hinkle (1995). The coudé-feed spectra were taken with $R=44000$. For the coudé-feed spectra, standard infrared reduction techniques were applied to two spectra taken at different positions along the slit. Velocities were then determined by cross-correlation of the spectra with velocity standards ( $\delta \mathrm{Oph}$ and $\alpha \mathrm{Cet}$ ) by means of the IRAF task fxcor. For a more detailed description of the data-reduction process see e.g. Hinkle et al. (2002). Typical uncertainty of those velocity measurements is $0.5 \mathrm{~km} \mathrm{~s}^{-1}$ (see e.g. Lebzelter et al. 2005).

\section{Light-curves}

When we started frequent velocity monitoring of WZCas we also attempted to obtain a simultaneous time series of highquality photometry in order to relate the velocity changes to the light changes. Unfortunately, owing to technical and other problems only three rather short parts of the light-curve were obtained (Fig. 1). Observations were made with the Austrian automatic photoelectric telescope (APT) located in southern Arizona. A detailed description of that instrument can be found in Strassmeier et al. (1997). Measurements were made in $V$ and $I$ filters of the Johnson/Cousins system. As comparison stars we used HD $224655(V-I=1.17)$ and HD $224940(V-I=1.10)$.

While the APT data are valuable in providing information on the colour change of the star over its variability period, longer and more complete light-curves are required for a comparison with the radial-velocity observations. The AAVSO and AFOEV data bases both contain large numbers of visual measurements of the brightness of WZ Cas. To reduce the scatter we first averaged all data from a single night and then took a running mean with a window size of seven data points. The AAVSO and AFOEV datasets were analyzed separately. Figure 2 shows the two resulting averaged light curves.

The most obvious variation visible in Fig. 2 is a period of about 1 year. We applied a Fourier analysis to both datasets independently with the program Period98 (Sperl 1998). The AAVSO data give a period of 364.6 days; the corresponding

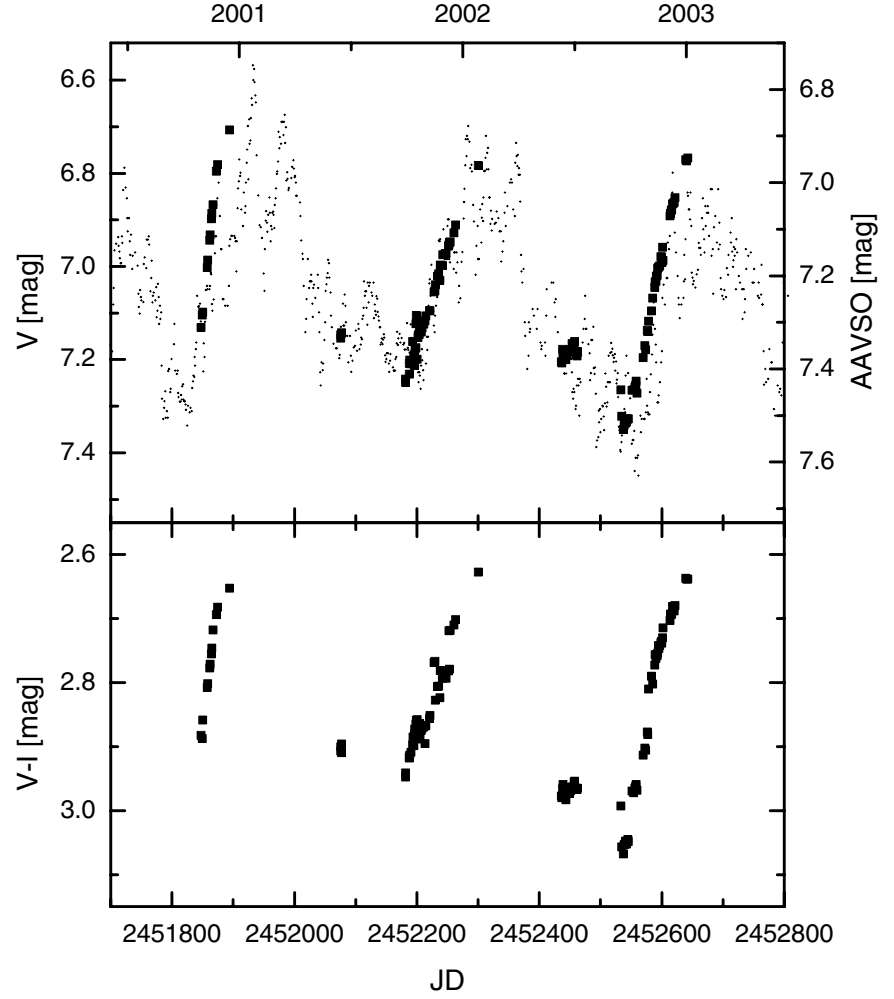

Fig. 1. APT measurements for WZCas. Top panel: $V$ light change. Large symbols are APT data, small dots indicate AAVSO data (see text). Visual and photometric data typically show an offset in magnitude (see the discussion in e.g. Lebzelter \& Kiss 2001). For an easier comparison the AAVSO data were scaled to the right-hand axis which is shifted slightly relatively to the left-hand axis (APT data). The shift is estimated from typical values (Lebzelter \& Kiss 2001) to give an approximate fit to the APT data and should not be taken as an accurate value. Bottom panel: variation in $V-I$.

value for the AFOEV data is 368.7 days. Those values are in tolerable agreement with one another, and also with the results of Kiss et al. (1999), who found from partly the same dataset a period of 373 days and a second one of 187 days. That second period also shows up in our analyses at 186.8 and 186.4 days, respectively. Variations on time-scales of a few tens of days also appear to be present in the AFOEV and AAVSO light curves. The light changes are clearly not strictly periodic, and thus confirm the star's classification as a semi-regular variable (SRV).

Inspection of the complete available light-curve, plotted in Fig. 3, shows intervals of large- and small-amplitude variation. These intervals do not seem to follow any periodicity. The points plotted with the heavier symbols in the years 1953-57 represent visual observations made by one of the present authors (R.F.G.); as in the cases of the other photometric comparisons shown in Figs. 1 and 2, there are systematic differences at certain times between those observations and the AAVSO ones, although there is agreement over the principal changes in magnitude.

Most of the time the agreement between different data sets is quite good, but in addition to the random errors typical of visual observations there are in several places considerable 

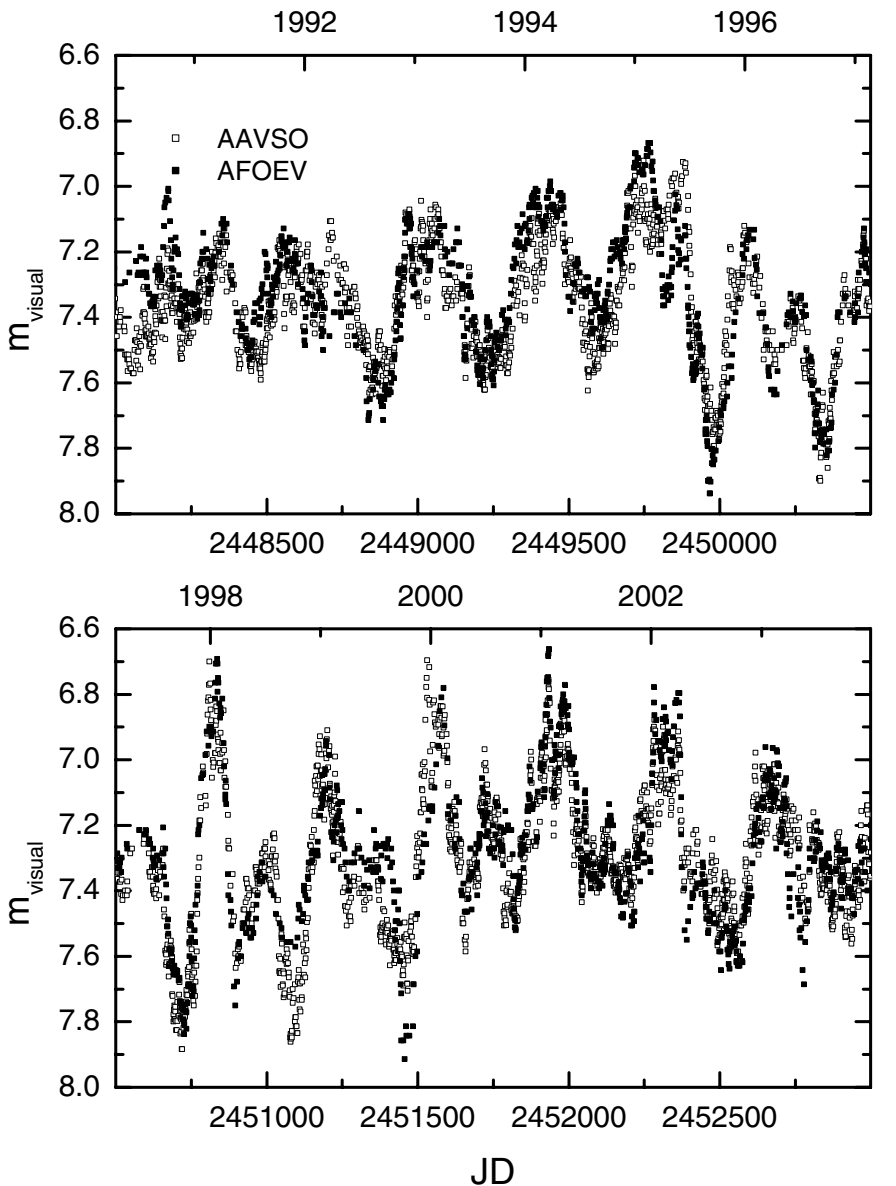

Fig. 2. Comparison of light-curve data from AAVSO (open symbols) and AFOEV (filled symbols). Both datasets were subjected to an averaging procedure as described in the text.

systematic discrepancies that occasionally reach several tenths of a magnitude, e.g. around 1998.75 and 2000.0. Analogous systematic discrepancies can be seen in certain places in the comparison of AAVSO with APT data in Fig. 1. WZ Cas is a difficult object for visual observers, owing to its extremely red colour and the lack of nearby comparison stars with anything approaching the same degree of redness. Part of the spread of visual results probably arises from actual differences between the sensitivities of different observers' eyes as a function of wavelength; another part can be due to the use of instruments of different apertures, bringing colour vision into play to a greater or lesser extent. Although, therefore, it must be accepted that visual photometry of a star as red as WZ Cas has its limitations, it is clear from the general agreement between the two independent sources of visual data plotted in Fig. 2 that those sources do provide a good impression of the major objective changes in the brightness of the star.

To check if the two identified periods are constant over time we applied a wavelet analysis to the whole AAVSO data set using the program WWZ provided by the AAVSO (Foster 1996). In Fig. 4 we show the resulting most probable period for each epoch. The one year period shows smooth variations with time but no cyclic behaviour can be seen. Over the time span of 55 years we see two maxima and three minima. These variations thus occur on time scales of several thousand days. The shorter period also shows significant changes with time but the change is less smooth than for the longer period. At some times this period seems to be hardly detectable.

\section{Recent variability of WZ Cas}

The more recent light curve (lower panel of Fig. 3) has two parts with a pulsation of relatively large amplitude, before JD 2445500 and after JD 2450000 , respectively, with a time of smaller-amplitude variations in between. Currently the amplitude seems to be decreasing again. In addition, the mean brightness of the star seems to vary slightly with time; again, no periodicity is obvious.

Velocity monitoring began at about JD 2445500 , i.e. towards the end of the first large-amplitude phase. In Fig. 5 we show part of our measurements from Coravel and near-infrared spectra on top of the AAVSO light-curve. Notice that the velocity axis has been inverted, and the scales have been arbitrarily adjusted with a view to demonstrating the essential similarity of the variations in the two parameters. Most of the time the velocity change nicely mirrors the light change. Such behaviour has been found in many semi-regular variables before (e.g. Lebzelter et al. 2000). Coravel and near-infrared data show a satisfying agreement. The typical peak-to-peak velocity range of the variation is approximately $6 \mathrm{~km} \mathrm{~s}^{-1}$. There is some indication in our data that between JD 2448000 and JD 2449500 , when the light amplitude was smaller, the velocity amplitude was also smaller, but owing to the low frequency of velocity monitoring at that epoch that result is somewhat uncertain.

After JD 2451500 we started a dense velocity monitoring (Fig. 6). The velocity changes are dominated by the one-year period and again largely mirror the light changes; light minima correspond to velocity maxima and vice versa. However, the amplitude of the velocity change is not well correlated with the amplitude in the light curve. The light-curve shows only weak cycle-to-cycle variation in the amplitude, while the velocity curve so far appears to show alternating strong and weak maxima. In the light change we find a tendency towards lower mean brightness from cycle to cycle, but that is not reflected in the velocity changes. The 186-d period found in the light-curve is not obvious in the radial velocities but it is in fact present and is retrieved by a Fourier analysis. The velocity amplitude in the more recent, densely observed, part of our dataset is the same as in the earlier part.

Since JD 2451500 we have in addition monitored the profile of the correlation dip produced in the Coravel observations. The dip looks like (and is in fact closely related to) the averaged profiles of a great many lines in the stellar spectrum. It is superimposed upon a flat continuum just like an isolated spectral line in a conventional plot of intensity against wavelength, but of course in the radial-velocity case the abscissae are in velocities. In Fig. 7 we show two examples of correlation dips. The area of the dip can be measured as an equivalent width, again with $\mathrm{km} \mathrm{s}^{-1}$ as the unit rather than the wavelength units used for spectral equivalent widths. The width of the dip reflects that of the spectral lines themselves, and for normal stars can be used as a measure of the stellar rotational velocity 

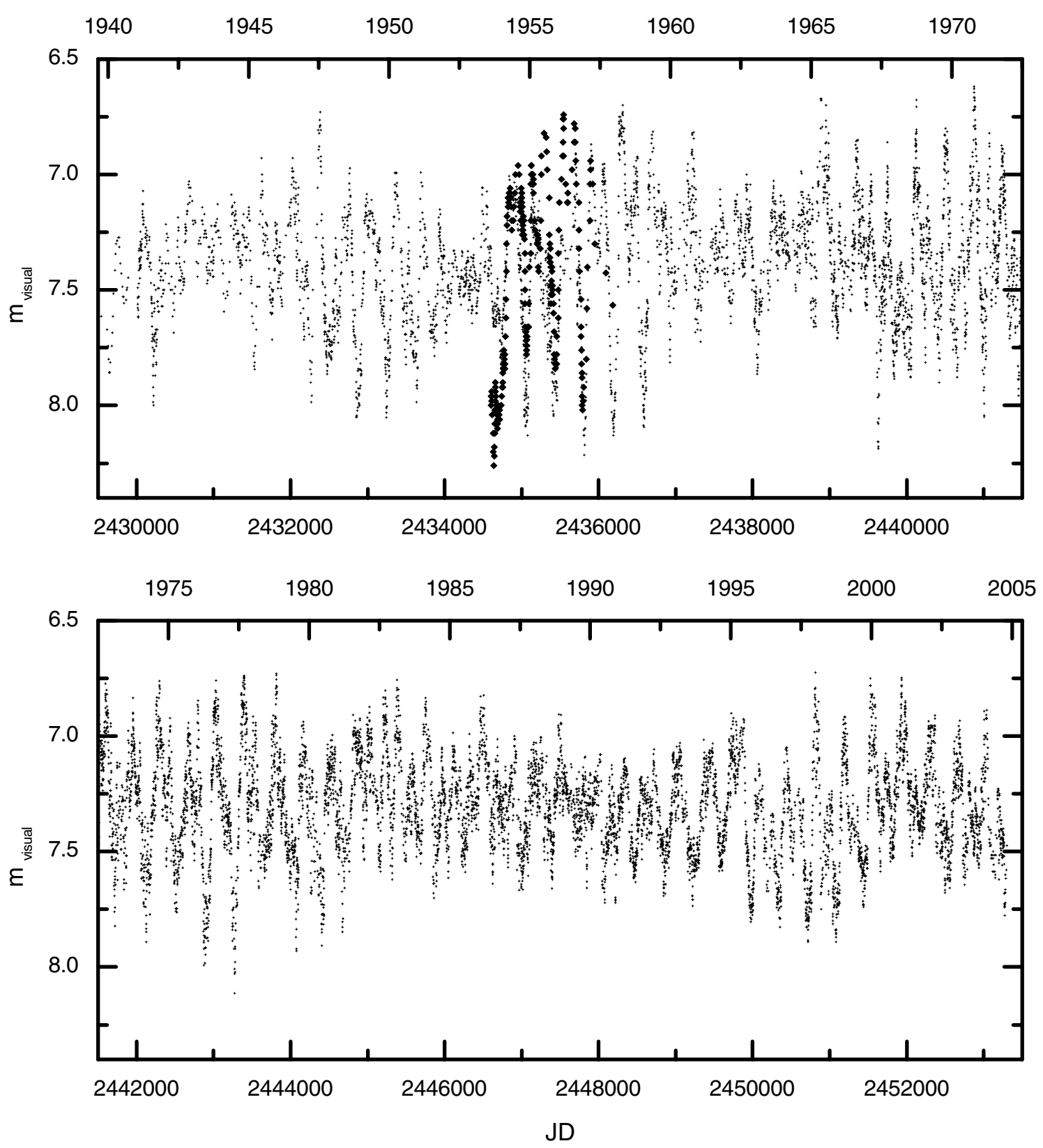

Fig. 3. The whole available light-curve of WZ Cas. Small dots show the measurements from the AAVSO database (see text), large dots indicate data obtained by RFG.

(Benz \& Mayor 1981). Lines that are significantly broadened by rotation have non-gaussian profiles, so we model rotational broadening by integrating the contributions of a lot of individual elements into which the area of a rotating, conventionally limb-darkened, stellar disc is conceptually divided. The initial profile, considered to correspond to zero rotation, is a noisefree version of the dips of minimum width given by many bright stars. We use two numbers to characterize the dip profile, equivalent width and $v \sin i$. The parameter that we call $v \sin i$ is just a dip-fitting parameter and should not necessarily be taken literally as a rotational velocity. The line broadening might well arise, at least in part, from turbulence and/or pulsation, as we discuss below.

The values of the two quantities are plotted against time in Fig. 8, which demonstrates that the correlation profile changed very significantly during our monitoring campaign. Besides some variation roughly related to the observed light and velocity changes ${ }^{1}$ there is a clear overall tendency towards lower values of the equivalent width and the apparent $v \sin i$ since the start of the observations: the correlation dip has narrowed significantly during that time span. That can also be seen in Fig. 7 where we compare the correlation dip obtained

1 As the photomultiplier in the Coravel instrument is not cooled, the dark count, which is a function of the ambient temperature, has some minor influence on the depth of the correlation profile. It is unfortunate that the period of the star is almost exactly one year, since seasonal temperature variations may mimic the same kind of variation in equivalent width. However, comparison with other sources suggests that that effect is clearly less than the periodic element in the observed variations. 


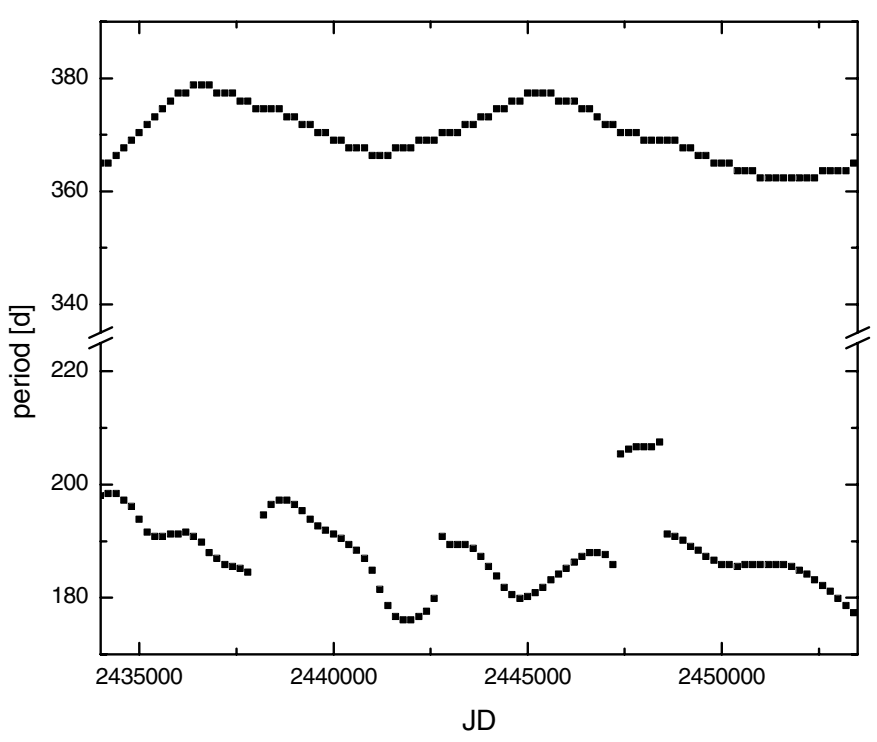

Fig. 4. Variability of the most probable period of WZ Cas with time from wavelet analysis. The values for the shorter period were derived from a separate analysis with a lower maximum period limit.

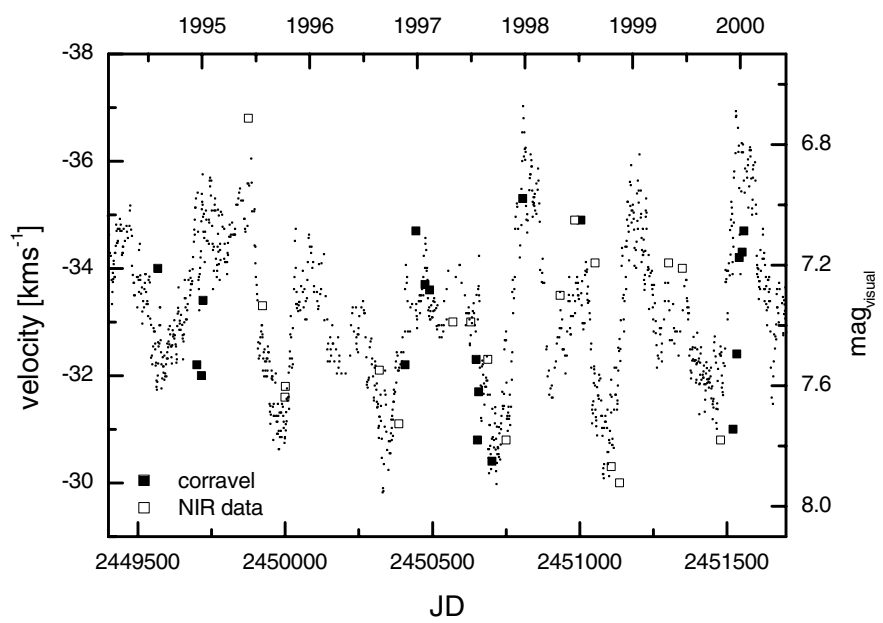

Fig. 5. First part of the velocity variations of WZCas on top of the AAVSO light change (small dots). Open boxes denote near-infrared measurements, filled boxes results from Coravel.

in December 1999 with the one from May 2003. Quite recently there was a remarkable rapid increase in the apparent $v \sin i$ value, i.e. the profile suddenly became wider again. Only a mild increase occurred in the equivalent width, and no special effect can be seen in the velocity curve.

\section{Additional high resolution spectra}

We managed to obtain one high-resolution spectrum of WZ Cas with the 2.2-m telescope at Calar Alto Observatory, on JD 2452895 , i.e. towards the end of our monitoring, when the star showed a rather low value of $v \sin i$. The spectrum was taken with the echelle spectrograph FOCES (Pfeiffer et al. 1998) in the visual range with a resolving power of 40000 . The spectrum was partly under-exposed, but it allowed measurement of the positions of several atomic lines. We identified from the atlas of carbon stars (Barnbaum 1994) 72 atomic lines between $\lambda \lambda 5600$ and $7800 \AA$, and measured

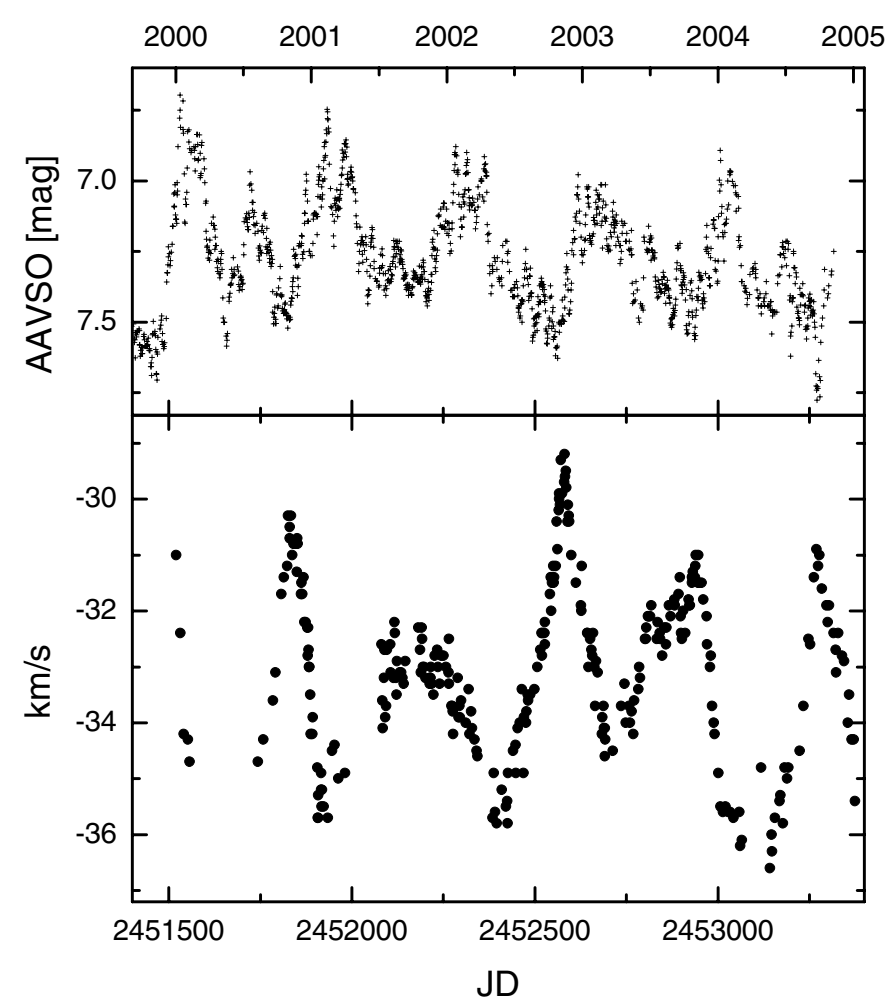

Fig. 6. Recent velocity variations of WZCas (bottom panel) compared with light variations.

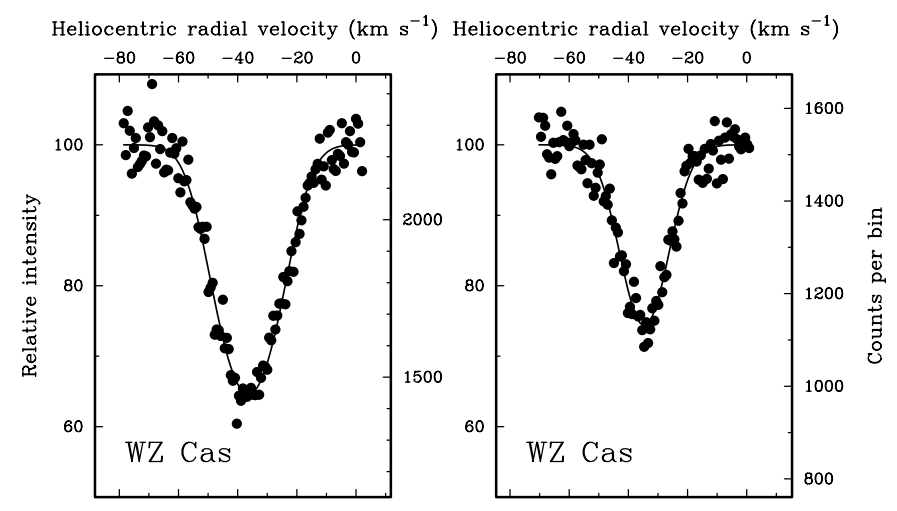

Fig. 7. Two examples illustrating the change of the correlation dip. Left panel: 1999 Dec. 28 (JD 2451 540). Right panel: 2003 May 24 (JD 2452783 ).

the velocity shift of the line centres. The mean velocity is $-32.3 \pm 1.9 \mathrm{~km} \mathrm{~s}^{-1}$, in excellent agreement with the findings from the Coravel measurements. The standard deviation gives no evidence of significant velocity differences between individual atomic lines. In addition to atomic lines, molecular lines of $\mathrm{CN}$ and $\mathrm{C}_{2}$ make a substantial contribution to the visual spectrum of WZ Cas. They form a dense forest, making it difficult to identify individual lines that are almost unblended. Ten lines most likely fulfilling that criterion were measured and gave the same velocity as the atomic lines.

A second optical-region high-resolution spectrum was kindly provided by Carlos Abia. It was obtained on 1997 December 8, i.e. quite some time before our dense monitoring started. A detailed description of that spectrum is given 


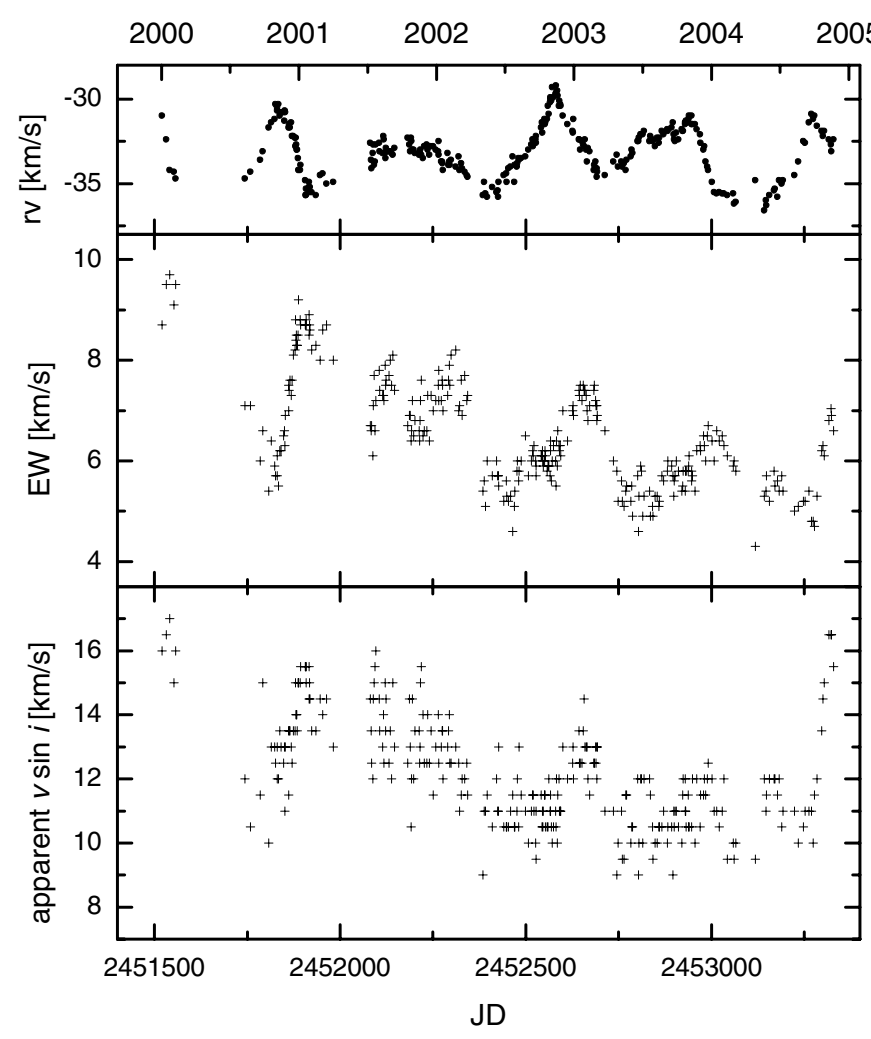

Fig. 8. Variations of the shape of the correlation dip with time. Middle panel: equivalent width. Bottom panel: apparent $v \sin i$. Top panel: velocity variations for comparison. See text for details.

in Abia \& Isern (2000). The resulting velocities were similar to those from our more recent spectrum. We also looked for differences in the widths or profiles of the lines, but no significant differences between the two spectra were found.

There is one excellent high-resolution near-infrared spectrum of the 1.6-2.4 $\mu \mathrm{m}$ region taken by Lambert et al. (1986). That spectrum has line profiles that are typical of the set of carbon stars observed in their survey. Lambert et al. derived a microturbulence of $2.2 \mathrm{~km} \mathrm{~s}^{-1}$, which is close to the mean value for their sample of 30 stars and suggests that WZ Cas is not very different from other carbon stars in that respect.

\section{Discussion}

The variability monitored over the last few years for WZCas exhibits various time-scales and does not affect the different tracers of variability equally. The principal time-scale is close to one year, a period that is obvious in both the photometric and the velocity data. A period about half as long is also present. In addition, we observe a longer-term variation in the width of the cross-correlation profile over a time-scale of $1000 \mathrm{~d}$ or so. Such a long-term variation in the line profiles has never been observed in an evolved single star before, but long-term photometric and velocity variations are known in late-type stars. A third level of variability is seen in the amplitude and mean brightness of the light-curve. The periodicities of the latter two are not clear, as any time-scales clearly exceed the length of our monitoring, but in the case of the amplitude change even more than 50 years of light-curve data do not reveal periodicity.

\subsection{The 366-d and 186-d variations}

Most AGB stars are radial pulsators (see e.g. Wood \& Sebo 1996, and references therein). By analogy with the behaviour of other AGB stars, we assume that the 366- and 186-day variations result from radial pulsation. The velocity variations of WZ Cas show an amplitude of a few $\mathrm{km} \mathrm{s}^{-1}$ and mirror the light variations, evidence that favours radial pulsation as an explanation. A period length of about a year, the main period of the radial-velocity variations, is typical of the C-rich AGB variables. The second period of about 186 days supports a model of radial pulsation as the primary origin of the photometric variations. The ratio of primary to secondary period in WZ Cas is approximately 2 . Such a value is to be expected if the two periods correspond to the fundamental and first-overtone modes (e.g. Wood et al. 1999). We therefore conclude that fundamental and first-overtone-mode radial pulsations are well suited to explain the main variation of the star.

According to a strict inversion of the Hipparcos parallax the star's $M_{\text {bol }}$ is at least 1 mag brighter than the value expected from the period-luminosity relationship for carbon-rich Miras (Groenewegen \& Whitelock 1996), but the uncertainty of the parallax is such that we cannot be sure that the over-luminosity is significant. As noted above, WZ Cas is a likely candidate to be unusually luminous because of having a higher mass than a typical C-rich variable. Hot bottom burning, which is indicated by the abundances, implies a mass of at least $4 M_{\odot}$.

\subsection{Changes in the width of the correlation profile}

The long-term change seen in the line profile has a time scale longer than the proposed fundamental mode of pulsation. Periods longer than the fundamental are known in AGB stars. For instance, the change in the line profile has a time-scale similar to long secondary periods (LSP) found for AGB stars by Wood et al. (2004). There are fundamental differences in these observations. The variation observed by us is a change in the correlation width of the line profile while Wood et al. (2004) observed mainly velocity changes. LSP remain a poorly understood phenomenon.

Summarizing the line-profile observations presented above, we see a general decline in the width of the correlation profile, expressed as $v \sin i$, with time over an interval of $\approx 1000 \mathrm{~d}$. Although it is not at all clear what is actually causing the changes we will use the expression $v \sin i$ for quantifying the width of the cross-correlation profile. There seems to be some kind of variation around the decline, which partly corresponds to variations seen in the radial-velocity change, but part of that variation seems to have no counterpart in the light or velocity variation. The equivalent width of the correlation-dip profile, which takes into account both the width and the depth of the dip, shows a similar trend at higher signal-to-noise.

The $v \sin i$ value of the correlation dip has undergone peakto-peak variations from 9 to $17 \mathrm{~km} \mathrm{~s}^{-1}$ over the course of our observations. After a bit of smoothing we might say that we saw a decrease of the $v \sin i$ value from about 14 to $10 \mathrm{~km} \mathrm{~s}^{-1}$ in a time span of $1000 \mathrm{~d}$. 


\subsubsection{Variation in rotational velocity}

There are a number of possible causes of the changes in the dip width. A naïve explanation is that there has been an actual change in $v \sin i$. Such a variation of the rotational velocity would require a change in radius of the star. To achieve a change in the rotational velocity from 14 to $10 \mathrm{~km} \mathrm{~s}^{-1}$, angular momentum of the surface layers being conserved, would require the radius to increase a factor of 1.4 , i.e. from perhaps 600 (see Sect. 1) to $840 R_{\odot}$. Alternatively the angular momentum could be supposed to change at constant radius.

Numerous objections obviously can be made to those scenarios. However, moderately rapid rotation of AGB stars is not impossible; it can not occur as the result of single-star evolution of a main-sequence rapid rotator, but can result from the merger of a binary system (Olivier \& Wood 2003). For a pulsating star the period of the pulsation is a monitor of the stellar radius. In the light of the discussion by Wood et al. (2004) we would expect to see a change in period length of about $66 \%$ if the stellar radius actually changed from 600 to $840 R_{\odot}$. Within the duration of the 1000-d trend, there is no indication of any change of that size in either the $366-\mathrm{d}$ or the $186-\mathrm{d}$ period. Therefore the star can not have changed its mean size by the required amount over the observed time span.

A binary scenario, affecting either rotation velocity or the rotation axis, might account for changes in the line profile. Again, the velocity curve (Figs. 5 and 6) provides no evidence of a companion. Similarly, the colours do not indicate a companion.

Differential rotation and the development of a magnetic cycle have been found plausible for AGB stars by several authors (e.g. Soker 2000; Blackman et al. 2001). For a simple estimate of the strength of magnetic field required to modify the surface rotation velocity of the star we calculate the field necessary for an Alfvén wave running through the whole star within the time-scales observed. Thereby we can use two different timescales for our estimate. In addition to the $\sim 1000$-d time-scale of the decline in line width, we see in Fig. 8 an abrupt increase in line width within about 45 days. Assuming a mean density in the stellar atmosphere of $10^{-8} \mathrm{~g} \mathrm{~cm}^{-3}$ (compare e.g. Bessell et al. 1989) we get for the shorter time-scale a required magnetic field of $3.8 \mathrm{G}$ and for the longer time-scale a field strength of $200 \mathrm{mG}$. Such field strengths do not seem impossible (compare Soker 2000 and Blackman et al. 2001). Most recently (e.g. Jordan et al. 2005, and references therein) observational evidence has been found for magnetic fields in the central stars of planetary nebulae. It is very likely that such magnetic fields are located mostly in the envelopes of those stars, where it has been conserved from earlier evolutionary phases. According to Jordan et al. (2005) only small to moderate - and thus possibly not observable - magnetic field strengths would be required during earlier evolutionary phases. From the time-scales involved, a magnetic cycle can not be excluded as a possibility. However, we stress that other observations directly supporting a magnetic cycle are lacking. The fields are far too small to be detected by Zeeman effects in the photospheric spectrum.

\subsubsection{Non-uniform surface}

Another possibility is that the star could have a spotted or otherwise non-uniform surface. Rotation could then indeed lead to a variation of the correlation profile. We can estimate a rotational period if we choose a radius of $600 R_{\odot}, \sin i \sim 1$ and a rotational velocity of $14 \mathrm{~km} \mathrm{~s}^{-1}$, giving about $2200 \mathrm{~d}$. If we use a velocity of $10 \mathrm{~km} \mathrm{~s}^{-1}$ we get a period of $3000 \mathrm{~d}$. Those are of very much the same order as the period of the longterm variation. An inhomogeneous surface does not necessarily have to stay constant, which could explain the lack of any periodicity of the long-term changes seen in the light-curve. One would expect, however, that asymmetry should be visible in the correlation dip as a spot or other azimuthal irregularity comes into the line of sight, but most of the time the dip has appeared symmetrical.

Clearly we are hampered by the lack of a time series of high-resolution spectra. The near-infrared spectra of carbon stars, unlike the visual spectra, are relatively uncrowded. However, contrast between the photosphere and star spots will be low in the infrared so it is likely that surface effects create a much larger effect in the optical.

Convective cells as non-uniform surface structure will be discussed separately below.

\subsubsection{Changes in the atmospheric conditions}

The available data do not allow us to decide on the possible effect on the correlation dip of a large velocity scatter between individual lines. Our high resolution optical spectrum was obtained close to the minimum value of $v \sin i$, when we would not need to invoke splitting into various velocity systems to explain the line-width. Unfortunately we do not have a spectrum obtained when the lines are known to have been wide. One would require two systems of lines (or lines split into two components) differing in velocity by at least $8 \mathrm{~km} \mathrm{~s}^{-1}$ to explain a change of $v \sin i$ by $4 \mathrm{~km} \mathrm{~s}^{-1}$. Such a change seems possible, by comparison with the values proposed for long-period variables by Alvarez et al. (2001). But the stars of their sample that appeared to show large variations typically also show much larger light variations accompanied by emission lines in their spectra (not observed for WZ Cas), indicating shock fronts running through their atmospheres. Furthermore we would expect some asymmetry in the correlation profiles, as illustrated in the paper by Alvarez et al., whereas most of the correlation dips of WZ Cas are rather symmetrical (Fig. 7).

Combining our spectrum with the older one provided by Abia it is not possible to reach any conclusion as to whether a velocity scatter is responsible for the observed variation, as we cannot relate the Abia spectrum to a Coravel dip profile. But as the width of the lines does not differ significantly between the two spectra, obviously the observed trend in the $v \sin i$ value must have reversed at some time.

Finally, the line-width may be changed by variations in macroturbulence. In general, late-type supergiants have lines broadened by macroturbulence. The broadening of spectral lines with decreasing gravity and increasing luminosity is apparent in high-resolution spectral atlases (e.g. Wallace \& Hinkle 1996). Gray \& Toner (1987) have shown by Fourier 
methods applied to high-resolution spectra how line-widths are to be apportioned between rotation and macroturbulence in a number of cases ranging in spectral types from $\mathrm{F}$ to $\mathrm{K}$ at luminosity class Ib; Bonsack \& Culver (1966) illustrated the consequences of attributing the whole of the line-width to macroturbulence. WZ Cas is a different type of star that can scarcely be expected to have a similar atmospheric structure, but it is a low-surface-gravity, high-luminosity object with a hugely extended and extremely tenuous atmosphere. It is perhaps possible that, without any significant change in the size of the star, a variation in line-width might be produced by a shift of the lineforming region. That could be tested only by a full dynamical modelling.

Several studies have dealt with the variation of macroturbulence in variable stars, mostly Cepheids (e.g. Stift \& Gillet 1994; Gillet et al. 1998, 1999). The turbulence variations in those cases are due to the global compression of the atmosphere during the pulsation. Accordingly they follow the light and velocity variations (compare Gillet et al. 1999). Changes in the equivalent width of the lines are observed and are also expected from pulsating models (Gillet et al. 1999). In the case of WZCas small variations in $v \sin i$ are correlated with radial velocity but there is not a correlation between the long term variations of $v \sin i$ and radial velocity (Fig. 8). Longer-period non-radial pulsations, and changes in the macroturbulence related to changing non-radial cells, are indeed possible.

\subsubsection{Convection}

Convection in red supergiants is dominated by a few convective cells (Schwarzschild 1975). Recent modelling in that field has been done by Freytag and collaborators (e.g. Freytag et al. 2002). In their recent paper (Freytag et al. 2005) they present line-profile variations arising from large convective cells in a red supergiant ( $\alpha$ Ori). They find roughly correlated variations both in the line depth and in the line width. The FWHM they measured from their synthetic line profiles varies by up to $10 \mathrm{~km} \mathrm{~s}^{-1}$. The variations occur on typical time-scales of half a year, but show no well expressed periodicity. Changes on shorter time-scales are also present. Interestingly, the velocity of the line core does not necessarily show significant changes with time in their models.

Those results seem to offer a plausible explanation for the observed variation in the $v \sin i$ value. While the current models suggest time-scales that are obviously too short for WZ Cas, additional adjustment of the model for specific properties of a carbon star (temperature, pulsation, etc. - Freytag's model was for an M Ia) would be of interest. If we simply transfer for the moment our measured $v \sin i$ variations into $F W H M$ variations of the spectral lines the amplitude of the modelled variation is in good agreement with our observations. Furthermore the lack of a strict periodicity and the possibility of a change without a major impact on the radial velocity tend to support that interpretation for WZ Cas. It looks also as if the relatively rapid variations seen at the end of our monitoring have a possible counterpart in the model results.

\subsection{Variations on a long time-scale}

Long-period light changes with a length of several thousand days have been reported for WZ Cas by Houk (1963). WZ Cas is listed by Houk with a long period of $4000 \mathrm{~d}$, but neither the reference listed by Houk nor the recent light change allow a true periodicity to be established. Very-long-period photometric and velocity changes are present in a large number of semiregular variables (e.g. Wood et al. 2004).

Unlike the cases of the stars studied by Wood et al. or Hinkle et al. (2002) the long-term variation of WZ Cas is not visible in the radial-velocity data. Hinkle et al. did not study the line profiles, while Wood et al. (2004) report variations in equivalent width of the $\mathrm{H} \alpha$ and the $\mathrm{NaD}$ line partly correlated with the long secondary period. Those lines, however, are not well suited for a comparison with our observations. According to Wood et al. the shape of the two lines is highly affected by the chromosphere and the mass loss region of the star, respectively, while most of the lines contributing to our correlation profile will be dominated by the conditions of the atmosphere. Neither the $\mathrm{H} \alpha$ nor $\mathrm{Na} \mathrm{D}$ line falls into the spectral range monitored by us, thus their specific variations do not contribute to our correlation profile. Therefore we cannot decide whether the line profile variations observed in WZCas and in the semiregular variables with long secondary periods investigated by Wood et al. have of the same origin. It is possible that the list of Houk (1963) includes stars with quite different origins for their respective long-term variabilities.

For the oxygen-rich semi-regular variable $\mathrm{L}^{2}$ Pup, Bedding et al. (2002) found the mean brightness to show a long-term dimming which they attributed to obscuration by dust. $\mathrm{L}^{2} \mathrm{Pup}$, however, has a mass-loss rate of $3 \times 10^{-7}$ to $5 \times 10^{-7} M_{\odot} \mathrm{yr}^{-1}$ (Jura et al.2002; Bedding et al.2002), i.e. by a factor of 50 to 100 more than WZCas. While dust formation must have some influence on the $V$ brightness of WZCas, we think that the current mass-loss rates can not have a major effect.

\section{Conclusions and outlook}

We have presented observations on the variability of the evolved carbon-rich star WZ Cas. Like most long-period variables it shows a complex behaviour of light and velocity changes. While we could attribute quite clearly the 366-d and 186-d variations to radial pulsation, the long-term variations on a time-scale of several thousand days remain an unsolved problem. Long-term variations are seen both in the lightcurve and in the line profiles but not in radial velocity. We have discussed several possibilities and shown that pulsation or an asymmetry of the star are unlikely origins for the observed long-term changes. The time-scales involved seem to allow an explanation in terms of a magnetic field or magnetic cycle, but there is no direct observational evidence for a magnetic field in WZ Cas.

A promising explanation of the line-width changes is offered by macroturbulence variations due to the development and movement of large convective cells. Models by Freytag (e.g. Freytag et al. 2005) exhibit variations, similar to those observed in WZCas, in the widths of synthetic line profiles. 
As all other interpretations discussed seem rather unlikely we favour convective motion in the atmosphere as the most probable explanation for the observed behaviour. We note that the motions of large convective cells are expected to have also a strong effect on the global structure of the magnetic field.

We suggest three observational tests. First, line-width measurements should be undertaken for stars with photometric periods exceeding the fundamental period. That would show whether the line-profile changes could be associated with strange pulsation modes (see Wood 2000). Evidence to date does not support that, but the coincidental agreement of the period lengths warrants additional investigation. Secondly, red supergiants, e.g. $\alpha$ Ori, should be monitored for variations in line width and depth. Red supergiants studied up to now (see above) have not shown such behaviour, but the phenomoenon may be highly temperature-sensitive and both WZCas and $\alpha$ Ori have effective temperatures decidedly lower than the stars previously studied. Thirdly, a detailed very-high-resolution study of the line profiles of $\mathrm{M}$ and $\mathrm{C}$ giants should be undertaken to investigate the shapes of the line profiles and the possible role of rotation versus convection.

Long-term photometric, radial-velocity, and line-profile monitoring of late-type stars is of interest. Previous investigations have shown that long-period variations are not a phenomenon limited to this single object but are very common among semi-regular variables. Detailed studies exist only for very few objects (e.g. Wood et al. 2004). Future developments of current models of large convective cells in red supergiants may offer an interesting interpretation of such long-term phenomena. The results presented here illustrate the value of further investigation of WZ Cas and similar stars.

Acknowledgements. Sincere thanks go to Ernst Dorfi and Martin Stift for very fruitful discussions. We wish to thank Cecilia Barnbaum for providing a list of $\mathrm{CN}$ lines. Thanks go also to Carlos Abia for providing an additional high-resolution spectrum of WZ Cas. T.L. has been supported by the Austrian Academy of Science (APART programme). APT observations have been made possible by the FWF-project P14365-PHY. We acknowledge with thanks the variable-star observations from the AAVSO International Database contributed by observers worldwide and used in this research. This research has made use of the AFOEV database, operated at CDS, France. R.F.G. is grateful to the Observatoire de Genève for the use of the OHP Coravel. Thanks go to the Director and staff of Calar Alto Observatory for taking one high-resolution spectrum of WZCas as a target of opportunity. Wavelet analysis was performed using the computer program WWZ, developed by the American Association of Variable Star Observers.

\section{References}

Abia, C., \& Isern, J. 2000, ApJ, 536, 438

Abia, C., Parlenko, \& de Laverny, P. 1999, A\&A, 351, 273

Alvarez, R., Jorissen, A., Plez, B., et al. 2001, A\&A, 379, 305

Barabier, M., Mayor, M., Mennessier, M. O., \& Petit, H. 1988, A\&AS, 72, 463

Baranne, A., Mayor, M., \& Poncet, J. L. 1979, Vistas Astron., 23, 279

Barnbaum, C. 1994, ApJS, 90, 317

Barnbaum, C., \& Hinkle, K. H. 1995, ApJ, 110, 805

Bedding, T., Zijlstra, A. A., Jones, A., et al. 2002, MNRAS, 337, 79

Benz, W., \& Mayor, M. 1981, A\&A, 93, 235
Bergeat, J., Knapik, A., \& Rutily, B. 2002, A\&A, 390, 967

Bessell, M. S., Brett, J. M., Scholz, M., \& Wood, P. R. 1989, A\&A, 213, 209

Blackman, E. G., Frank, A., Markiel, J. A., Thomas, J. H., \& van Horn, H. M. 2001, Nature, 409, 485

Bonsack, W. K., \& Culver, R. B. 1966, ApJ, 145, 767

Fletcher, J. M., Harris, H. C., McClure, R. D., \& Scarfe, C. D. 1982, PASP, 94, 1017

Foster, G. 1996, AJ, 112, 1709

Freytag, B., Steffen, M., \& Dorch, B. 2002, AN, 323, 213

Freytag, B., Mizuno-Wiedner, M., \& Gustafsson, B. 2005, in 13th Workshop on Cool Stars, Stellar Systems, and the Sun, ed. F. Favata, ESA-SP, in press

Gautschy-Loidl, R., Höfner, S., Jorgensen, U. G., \& Hron, J. 2004, A\&A, 422, 289

Gillet, D., Debieve, J. F., Fokin, A. B., \& Mazauric, S. 1998, A\&A, 332,235

Gillet, D., Fokin, B., Breitfellner, M. G., Mazauric, S., \& Nicolas, A. 1999, A\&A, 344, 935

Gray, D. F., \& Toner, C. G. 1987, ApJ, 322, 360

Griffin, R. F. 1967, ApJ, 148, 465

Griffin, R. F., \& Gunn, J. E. 1974, ApJ, 191, 545

Groenewegen, M. A. T., \& Whitelock, P. A. 1996, MNRAS, 281, 1347

Jordan, S., Werner, K., \& O'Toole, S. J. 2005, A\&A, in press

Jura, M., Chen, C., \& Plavchan, P. 2002, ApJ, 569, 964

Herbig, G. H. 1955, PASP, 67, 181

Houk, N. 1963, AJ, 68, 253

Joyce, R. R., Hinkle, K. H., Meyer, M. R., \& Skrutskie, M. F. 1998, Proc. SPIE, 3354, 741

Hinkle, K. H., Lebzelter, T., Joyce, R. R., \& Fekel, F. C. 2002, AJ, 123,1002

Keenan, P. C. 1993, PASP, 105, 905

Kiss, L. L., Szatmary, K., Cadmus, R. R., Jr., \& Mattei, J. A. 1999, A\&A, 346, 542

Lambert, D. L., Gustafsson, B., Eriksson, K., \& Hinkle, K. H. 1986, ApJS, 62, 373

Lebzelter, T., \& Kiss, L. L. 2001, A\&A, 380, 388

Lebzelter, T., Kiss, L. L., \& Hinkle, K. H. 2000, A\&A, 361, 167

Lebzelter, T., Hinkle, K. H., Wood, P. R., Joyce, R. R., \& Fekel, F. C. 2005, A\&A, 431, 623

Olivier, E. A., \& Wood, P. R. 2003, ApJ, 584, 1035

Olofsson, H., Eriksson, K., Gustafsson, B., \& Carlstroem, U. 1993, ApJS, 87, 305

Pfeiffer, M. J., Frank, C., Baumueller, D., Fuhrmann, K., \& Gehren, T. 1998, A\&AS, 130, 381

Richichi, A., \& Percheron, I. 2002, A\&A, 386, 492

Schwarzschild, M. 1975, ApJ, 195, 137

Schöier, F. L., \& Olofsson, H. 2001, A\&A, 368, 969

Soker, N. 2000, ApJ, 540, 436

Sperl, M. 1998, CoAst, 111, 1

Stift, M. J., \& Gillet, D. 1994, in Eighth Cambridge Workshop on Cool Stars, Stellar Systems, and the Sun, ed. J. P. Caillault, ASP Conf. Ser., 64, 735

Strassmeier, K. G., Boyd, L. J., Epand, D. H., \& Granzer, T. 1997, PASP, 109, 697

van Belle, G. T., Thompson, R. R., \& Creech-Eakman, M. J. 2002, AJ, 124, 1706

Wallace, L., \& Hinkle, K. H. 1996, ApJS, 107, 312

Wood, P. R., and the MACHO Collaboration 1999, in Asymptotic Giant Branch Stars, ed. T. Le Bertre, A. Lèbre, \& C. Waelkens (San Francisco: ASP), IAU Symp., 191, 151

Wood, P. R. 2000, PASA, 17, 18

Wood, P. R., Olivier, E., \& Kawaler, S. 2004, ApJ, 604, 800

Wood, P. R., \& Sebo, K. M. 1996, MNRAS, 282, 958 